\title{
LETTER
}

\section{Clinical features and development of sepsis in patients infected with SARS-CoV-2: a retrospective analysis of 150 cases outside Wuhan, China}

\author{
Di Ren ${ }^{1,2}$, Chao Ren ${ }^{3}$, Ren-qi Yao ${ }^{3}$, Yong-wen Feng ${ }^{1 *}$ and Yong-ming Yao ${ }^{1,3^{*}}$ (])
}

(๑) 2020 Springer-Verlag GmbH Germany, part of Springer Nature

Dear Editor,

The outbreak of novel coronavirus disease (COVID-19) that began in December 2019 has posed a great threat to human health and been declared a global pandemic by the World Health Organization [1-3]. Shenzhen, an important and special economic zone in China, shares a large floating population with Hubei province. From the first occurrence of COVID-19 on January 11, 2020, to April 26, 2020, there were 461 cases confirmed with infection of severe acute respiratory syndrome coronavirus 2 (SARS-CoV-2), including 12 patients who remained in the hospital, 3 deaths, and 446 discharged patients [4]. In the present study, we aimed to describe the clinical characteristics of COVID-19 patients in Shenzhen and identify risk factors for the development of SARS-CoV2-induced sepsis in imported COVID-19 patients.

In this retrospective study, patients who were confirmed to have SARS-CoV-2 infection and admitted to the Third People's Hospital of Shenzhen from January 11 to February 12, 2020, were enrolled. Clinical data were extracted and followed up to March 11, 2020, by using predesigned data collection forms. The baseline characteristics of all enrolled patients in the sepsis and

\footnotetext{
*Correspondence: fengyongwen2008@126.com; c_ff@sina.com ${ }^{1}$ Department of Critical Care Medicine, The Second People's Hospital of Shenzhen, 3002 Sungang West Road, Futian District, Shenzhen 518035, People's Republic of China

${ }^{3}$ Trauma Research Center, Fourth Medical Center and Medical Innovation Research Department of the Chinese PLA General Hospital, 51 Fucheng Road, Haidian District, Beijing 100048, People's Republic of China Full author information is available at the end of the article Di Ren, Chao Ren and Ren-qi Yao have contributed equally to this manuscript.
}

non-sepsis groups were summarized and compared by applying Student's $t$ test, the Chi-square test, Fisher's exact test, and the Mann-Whitney $U$ test as appropriate. Continuous variables were presented as the mean (standard deviation [SD]) or median (interquartile range $[\mathrm{IQR}])$, while categorical or ranked data were reported as counts and proportions.

A total of 150 hospitalized COVID-19 patients were enrolled in this study, including 49 (32.7\%) patients with SARS-CoV-2-induced sepsis at hospital admission and 101 (67.3\%) non-septic patients (Table 1). Patients with viral sepsis were much older than those without sepsis (63 vs. 46 years, $P<0.001$ ) and presented with more comorbidities, including hypertension (14 [28.6\%] vs. 11 [10.9\%], $P=0.006)$ and diabetes (9 [18.4\%] vs. 3 [3\%], $P=0.003)$. Septic patients had significantly higher neutrophil counts, monocyte counts, international normalized ratios, D-dimer values, alanine aminotransferase, aspartate aminotransferase, serum creatinine, blood urea nitrogen, creatine kinase, lactate dehydrogenase, prothrombin times and activated partial thromboplastin times than non-septic patients, but their lymphocyte counts, platelet counts, and albumin levels were significantly lower. Septic patients were more likely to be transferred to the ICU (28 [57.1\%] vs. 10 [9.9\%]; $P<0.001)$ and had a significantly prolonged hospital stay (median days, 23.5 days [IQR, 16.3-32.8] vs. 15 days [IQR, 13-20]; $P<0.001)$ than non-septic patients. Additionally, deaths (3 [6.1\%]) occurred solely among patients who developed sepsis at hospital admission. Exposure history, platelet count, $\mathrm{T}$ lymphocyte count, cytotoxic T lymphocyte count, IL-6,

\section{实


Table 1 Baseline characteristics of 150 patients confirmed with COVID-19 in Shenzhen, China

\begin{tabular}{|c|c|c|c|c|}
\hline & \multicolumn{4}{|c|}{ No. $(\%)$ of patients } \\
\hline & Total $(n=150)$ & Sepsis $(n=49)$ & Non-sepsis $(n=101)$ & $P$ value \\
\hline \multicolumn{5}{|l|}{ Demographic characteristics } \\
\hline Age, median (IQR), years & $54(37-63)$ & $63(55.5-66)$ & $46(34-57)$ & $<0.001$ \\
\hline Female & $68(45.3)$ & $17(34.7)$ & $51(50.5)$ & 0.068 \\
\hline BMI, mean (SD) & $23.7(3.7)$ & $25.0(3.3)$ & $23.1(3.8)$ & 0.004 \\
\hline Causes of infection & & & & 0.005 \\
\hline Traveling history of Hubei province & $133(88.7)$ & $38(77.6)$ & $95(94.1)$ & \\
\hline Contact with local confirmed cases & $1(0.7)$ & $1(2)$ & $0(0)$ & \\
\hline Undetermined cause of infection & $16(10.6)$ & $10(20.4)$ & $6(5.9)$ & \\
\hline \multicolumn{5}{|l|}{ Comorbidities } \\
\hline Hypertension & $25(16.7)$ & $14(28.6)$ & $11(10.9)$ & 0.006 \\
\hline Diabetes & $12(8)$ & $9(18.4)$ & $3(3)$ & 0.003 \\
\hline Coronary heart disease & $8(5.3)$ & $4(8.2)$ & $4(4)$ & 0.492 \\
\hline Chronic bronchitis & $4(2.7)$ & $2(4.1)$ & $2(2)$ & 0.835 \\
\hline Smoking history & $3(2)$ & $3(6.1)$ & $0(0)$ & 0.059 \\
\hline Malignant tumor & $3(2)$ & $2(4.1)$ & $1(1)$ & 0.518 \\
\hline Gout & $3(2)$ & $2(4.1)$ & $1(1)$ & 0.518 \\
\hline Cerebrovascular disease & $2(1.3)$ & $2(4.1)$ & $0(0)$ & 0.105 \\
\hline Immunocompromised diseases & $1(0.7)$ & $0(0)$ & $1(1)$ & $>0.99$ \\
\hline \multicolumn{5}{|l|}{ Signs and symptoms } \\
\hline Fever & $125(83.3)$ & $42(85.7)$ & $83(82.2)$ & 0.586 \\
\hline Dry cough & $44(29.3)$ & $16(32.7)$ & $28(27.7)$ & 0.534 \\
\hline Expectoration & $35(23.3)$ & $14(28.6)$ & $21(20.8)$ & 0.291 \\
\hline Fatigue & $33(22)$ & $16(32.7)$ & $17(16.8)$ & 0.028 \\
\hline Myalgia & $32(21.3)$ & $13(26.5)$ & $19(18.8)$ & 0.279 \\
\hline Chest distress & $13(8.7)$ & $6(12.2)$ & $7(6.9)$ & 0.438 \\
\hline Dizziness & $11(7.3)$ & $7(14.3)$ & $4(4)$ & 0.052 \\
\hline Headache & $10(6.7)$ & $3(6.1)$ & $7(6.9)$ & $>0.99$ \\
\hline Anorexia & $10(6.7)$ & $6(12.2)$ & $4(4)$ & 0.119 \\
\hline Diarrhea & $10(6.7)$ & $4(8.2)$ & $6(5.9)$ & 0.871 \\
\hline Nausea & $5(3.3)$ & $4(8.2)$ & $1(1)$ & 0.070 \\
\hline Dyspnea & $4(2.7)$ & $2(4.1)$ & $2(2)$ & 0.835 \\
\hline Stomachache & $2(1.3)$ & $1(2)$ & $1(1)$ & 0.548 \\
\hline Vomiting & $1(0.7)$ & $0(0)$ & $1(1)$ & $>0.99$ \\
\hline No signs and symptoms & $10(6.7)$ & $2(4.1)$ & $8(7.9)$ & 0.593 \\
\hline Body temperature, median (IQR), ${ }^{\circ} \mathrm{C}$ & $37.2(36.7-38)$ & $37.8(37.2-38.3)$ & $36.9(36.7-37.8)$ & $<0.001$ \\
\hline Heart rates, median (IQR), /min & $89.5(83-98)$ & $93(86-101)$ & $88(81-96)$ & 0.025 \\
\hline Respiratory rates, median (IQR), /min & $20(19-21)$ & $21(20-22.5)$ & $20(19-20)$ & $<0.001$ \\
\hline Mean arterial pressure, median (IQR), mmHg & $95(89.7-103.4)$ & $96.7(91.8-108.8)$ & $94.3(88.8-102.8)$ & 0.072 \\
\hline Diastolic blood pressure, median (IQR), mmHg & $80(74.8-89)$ & $82(75-88)$ & $79(74-89)$ & 0.432 \\
\hline Systolic blood pressure, median (IQR), $\mathrm{mmHg}$ & $128(118-139)$ & $128(123.5-152)$ & $126(117-138)$ & 0.025 \\
\hline APACHE II, median (IQR) & $4(2-6)$ & $6(4-8.5)$ & $3(1-5.5)$ & $<0.001$ \\
\hline SOFA, median (IQR) & $1(0-2)$ & $2(2-3)$ & $0(0-1)$ & $<0.001$ \\
\hline Onset of symptoms to hospital admission, median (IQR), days & $4(2-7)$ & $4(2-7.5)$ & $3(2-5.5)$ & 0.044 \\
\hline \multicolumn{5}{|l|}{ Complications } \\
\hline ARDS & $87(58)$ & $47(95.9)$ & $40(39.6)$ & $<0.001$ \\
\hline Acute liver injury & $28(18.7)$ & $20(40.8)$ & $8(7.9)$ & $<0.001$ \\
\hline Acute kidney injury & $11(7.3)$ & $10(20.4)$ & $1(1)$ & $<0.001$ \\
\hline Acute cardiac injury & $11(7.3)$ & $8(16.3)$ & $3(3)$ & 0.009 \\
\hline
\end{tabular}


Table 1 (continued)

\begin{tabular}{|c|c|c|c|c|}
\hline & \multicolumn{4}{|c|}{ No. $(\%)$ of patients } \\
\hline & Total $(n=150)$ & Sepsis $(n=49)$ & Non-sepsis $(n=101)$ & $P$ value \\
\hline Shock & $9(6)$ & $7(14.3)$ & $2(2)$ & 0.009 \\
\hline Coagulopathy & $9(6)$ & $9(18.4)$ & $0(0)$ & $<0.001$ \\
\hline Secondary infection & $17(11.3)$ & $9(18.4)$ & $8(7.9)$ & 0.058 \\
\hline \multicolumn{5}{|l|}{ Prognosis } \\
\hline Discharge from hospital & $126(84)$ & $36(73.5)$ & $90(89.1)$ & 0.014 \\
\hline Length of stay in hospital, median (IQR), days & $16(13-24.3)$ & $23.5(16.3-32.8)$ & $15(13-20)$ & $<0.001$ \\
\hline Hospital admission to ICU admission, median (IQR), days & $6(2-9)$ & $5(2-8.8)$ & $7(4.8-9.3)$ & 0.434 \\
\hline ICU admission & $38(25.3)$ & $28(57.1)$ & $10(9.9)$ & $<0.001$ \\
\hline Length of stay in ICU, median (IQR), days & $7(4-16)$ & $9(4-19.8)$ & $4(2-11)$ & 0.192 \\
\hline In-hospital death & $3(2)$ & $3(6.1)$ & $0(0)$ & 0.059 \\
\hline
\end{tabular}

Data were presented as median (IQR) or mean (SD). $\mathrm{n}(\%)$ referred to the total number of patients with available data

ICU Intensive care unit, IQR Interquartile range, SD Standard deviation, BMI Body mass index, APACHE II Acute physiology and chronic health evaluation II, SOFA Sequential organ failure assessment, ARDS Acute respiratory distress syndrome

$P$ values indicated differences between sepsis and non-sepsis patients, in which $P<0.05$ was deemed as statistical significance

serum creatinine, erythrocyte sedimentation rate, and sodium might be useful for predicting the incidence of SARS-CoV-2-infection-induced sepsis (electronic supplementary materials).

In conclusion, patients with SARS-CoV-2 infection are likely to develop sepsis at hospital admission, which are characterized by failed homeostasis between the innate and adaptive immune responses partly due to the loss of lymphocytes. The development of sepsis might be associated with greater organ dysfunction and worse outcomes in this small cohort of patients from Shenzhen.

\section{Electronic supplementary material}

The online version of this article (https://doi.org/10.1007/s00134-020-06084-5) contains supplementary material, which is available to authorized users.

\section{Author details}

1 Department of Critical Care Medicine, The Second People's Hospital of Shenzhen, 3002 Sungang West Road, Futian District, Shenzhen 518035, People's Republic of China. ${ }^{2}$ Department of Critical Care Medicine, The Third People's Hospital of Shenzhen, Shenzhen 518020, People's Republic of China. ${ }^{3}$ Trauma Research Center, Fourth Medical Center and Medical Innovation Research Department of the Chinese PLA General Hospital, 51 Fucheng Road, Haidian District, Beijing 100048, People's Republic of China.

\section{Acknowledgements}

We thank all patients included in this study, and gratefully acknowledge all the front-line physicians in fighting against SARS-CoV-2 infection. We greatly appreciate the works from Prof. Jin-xiu Li from the Department of Critical Care Medicine, the Third People's Hospital of Shenzhen, Prof. Ying Li from the Department of Critical Care Medicine, The Second People's Hospital of Shenzhen, Prof. Xue-yan Liu from the Department of Critical Care Medicine, The People's Hospital of Shenzhen, Prof. Lei Huang from the Department of Critical Care Medicine, Peking University Shenzhen Hospital, Prof. Yong Liu from the Department of Critical Care Medicine, Shenzhen Hospital of Southern Medical University, Prof. Mian Peng from the Department of Critical Care Medicine, The Third Affiliated Hospital of Shenzhen University, and Dr. Yao Yao from the Center for Healthy Aging and Development Studies, National School of Development, Peking University in recruiting patients and providing technical supports.

\section{Author's contributions}

YWF and YMY contributed equally to this paper and were joint corresponding authors. DR, CR, RQY were joint first authors. All corresponding and first authors contributed to study concept and design. DR extracted epidemiological and clinical data. CR and RQY performed the statistical analyses. $\mathrm{DR}, \mathrm{CR}$ and RQY co-drafted the initial version of manuscript. All authors provided critical revision of the manuscript and approved the final draft for publication. YWF was responsible for the integrity and accuracy of the data and was the guarantor. The corresponding authors attest that all listed authors meet authorship criteria and that no others meeting the criteria have been omitted.

\section{Funding}

This work was supported by the National Natural Science Foundation of China ( 81730057 by YMY, 81801935 by CR), Sanming Project of Medicine in Shenzhen (SZSM20162011 by YMY and YWF), and the Military Medical Innovation Program of Chinese PLA (18CXZ026 by YMY).

\section{Compliance with ethical standards}

\section{Conflicts of interest}

The authors declare that they have no conflict of interest.

\section{Ethical approval}

This study was approved by the Committee on the Ethics of Medicine, the Second People's Hospital of Shenzhen (20200224002), China.

\section{Publisher's Note}

Springer Nature remains neutral with regard to jurisdictional claims in published maps and institutional affiliations.

\section{Accepted: 2 May 2020}

Published online: 15 May 2020

\section{References}

1. World Health Organization (2020) Coronavirus disease (COVID-2019) situation reports: situation report-97. https://www.who.int/docs/defau It-source/coronaviruse/situation-reports/20200426-sitrep-97-covid-19. pdf?sfvrsn = d1c3e800_6. Accessed 26 April 2020

2. Cucinotta D, Vanelli M (2020) WHO declares COVID-19 a pandemic. Acta Biomed 91 (1):157-160. https://doi.org/10.23750/abm.v91i1.9397 
3. Wang D, Hu B, Hu C, Zhu F, Liu X, Zhang J, Wang B, Xiang H, Cheng Z, Xiong Y, Zhao Y, Li Y, Wang X, Peng Z (2020) Clinical characteristics of 138 hospitalized patients with 2019 novel coronavirus-infected pneumonia in Wuhan, China. JAMA 1:2-8. https://doi.org/10.1001/jama.2020.1585
4. Shenzhen Municipal Health Commission (2020) Updates on COVID-19 cases in Shenzhen (April 26). http://wjw.sz.gov.cn/yqxx/202004/t2020 0426_19190314.htm. Assessed 26 April 2020 